\title{
HIV Seroprevalence Among Long-Stay Patients in a State Psychiatric Hospital
}

Ilan Meyer, M.A., M.Phil. Karen McKinnon, M.A. Francine Cournos, M.D. Maureen Empfield, M.D. Sam Bavli, M.D.

Diane Engel, M.S.W. Alan Weinstock, M.S., M.P.A.

An elevated rate of HIV infection among new admissions to psychiatric hospitals is now well established, with rates reported in anonymous surveys ranging between 5.2 percent (1) and 7.1 percent (2). However, no studies have reported HIV seroprevalence among long-stay psychiatric patients.

We investigated this question at a state psychiatric hospital in the borough of Queens in New York City. We expected that long-stay patients would have significantly lower rates of HIV infection than new admissions due to greater disability and fewer opportunities for sexual and drug use risk behaviors in the community.

A sizable proportion of psychiatric patients still remain continuously hospitalized (3). Unfortunately, the prevalence of sexual and drug use risk behaviors among these patients is not well established. Long-stay psychiatric inpatients may have less alcohol and drug abuse than short-stay (4) and "revolving-door" (5) patients.

Assessing the incidence of sexual activity on inpatient units is difficult. A one-year study of mixed-gender intensive treatment units found

The authors are affiliated with the New York State Office of Mental Health. Address correspondence to Dr. Cournos at the New York State Psychiatric Institute, 722 West 168 Street, New York, New York 10032. that 16 of 1,060 patients ( 1.5 percent) had engaged in overt heterosexual activity while hospitalized (6). In contrast, a study of HIV risk behavior among severely ill state hospital psychiatric inpatients and outpatients found that more than 50 percent reported sexual activity in the past six months (Cournos F, McKinnon K, Meyer-Bahlburg $\mathrm{H}$, et al, unpublished manuscript, 1992).

Long-term units, especially those with low staff-patient ratios, may have higher rates of sexual activity among patients than better-staffed acute-care units, with sex occurring either on the ward or in secluded areas on hospital grounds. It is unclear to what extent sexual activity is underestimated and concealed within hospitals $(7,8)$ and to what extent institutionalization is a significant barrier to sexual activity (9), although staff on long-stay wards appear to view patients' sexual activity as unavoidable (8).

\section{Methods}

Any patient at Creedmoor Psychiatric Center whose admission anniversary occurred between April 9 and July 31,1990 , and who was between the ages of 18 and 59 was included in this study $(\mathrm{N}=236)$. All patients had been in the hospital at least one year. Creedmoor does not admit patients whose primary diagnosis is substance abuse or dementia.

Following current federal regulations for anonymous sampling, we obtained discarded blood from samples drawn for a routine annual medical examination. This procedure has been described elsewhere (1). Patients' identification number, date of birth, admission diagnosis, number of previous hospitalizations, and current length of stay were obtained from their charts to generate a group profile unlinked to their HIV status.
Patients' age, gender, ethnicity, and known history of male homosexual activity and use of injected drugs were obtained from charts and linked to antibody results using a special code to preserve anonymity. The few Asian patients on the hospital census were grouped with Caucasians to preserve anonymity (1).

Blood specimens were not collected from 37 eligible long-stay patients for the following reasons: the patient was discharged or eloped before the blood draw, the patient refused to allow blood to be drawn, an insufficient quantity of blood was collected, and the annual examination was delayed more than 30 days beyond the end of the study period. The only significant diagnostic difference between the 199 patients whose blood we tested and the 37 patients whose blood we did not obtain $\left(\chi^{2}=8.97, \mathrm{df}=3, \mathrm{p}=.03\right)$ was that untested patients were diagnosed more frequently as having psychotic disorder not otherwise specified (13.5 percent versus 4 percent) or organic disorder (10.8 percent versus 4 percent). However, since there is no reason to believe that these diagnoses are related to increased risk for HIV, and since no patient in either group had AIDS-related or other dementia noted in the chart, we do not believe these differences are likely to have biased our findings.

Of the 199 tested patients, 140 patients ( 70.4 percent) were diagnosed as having schizophrenia, 12 had schizoaffective disorder (6 percent), eight had other psychotic disorders ( 4 percent), 17 had mood disorders (8.6 percent), eight had organic disorders (4 percent), and 14 had other psychiatric disorders ( 7 percent). Ten of the 199 tested patients ( 5 percent) had either no previous psychiatric hospitalization or one such hospitalization, 159 patients (79.9 percent) had from two to five hospitalizations, and 30 patients (15.1 percent) had more than five. The median length of the current hospital stay was 38 months (mean \pm $\mathrm{SD}=62.10 \pm 72.03$ months, range $=$ 12.5 to 615 months).

Specimens were tested for HIV-1 antibodies by enzyme immunoassay. Reactive specimens were tested with 
Table 1

Association of HIV infection, demographic characteristics, homosexual activity among men, and injected drug use among 199 long-stay psychiatric inpatients

\begin{tabular}{|c|c|c|c|c|c|c|}
\hline \multirow[b]{2}{*}{ Characteristic } & \multirow{2}{*}{$\begin{array}{l}N \text { rested } \\
\text { for HIV }\end{array}$} & \multicolumn{2}{|c|}{$\begin{array}{l}\text { HIV } \\
\text { positive }\end{array}$} & \multirow{2}{*}{$\begin{array}{l}\text { Unadjust- } \\
\text { ed odds } \\
\text { ratio }\end{array}$} & \multirow{2}{*}{$\begin{array}{l}95 \% \text { con- } \\
\text { fidence } \\
\text { interval }\end{array}$} & \multirow[b]{2}{*}{$\mathrm{p}$} \\
\hline & & $\mathbf{N}$ & $\%$ & & & \\
\hline Age & & & & 1.39 & .34 to 5.71 & .65 \\
\hline 18 to 39 & 115 & 4 & 3.5 & & & \\
\hline 40 to 59 & 84 & 4 & 4.8 & & & \\
\hline Ethnicity ${ }^{1}$ & & & & 2.84 & .56 to 14.44 & .21 \\
\hline Black & 73 & 3 & 4.1 & & & \\
\hline Hispanic & 31 & 3 & 9.7 & & & \\
\hline Caucasian or & & & & & & \\
\hline Asian & 95 & 2 & 2.1 & & & \\
\hline Gender & & & & 1.16 & .27 to 5.02 & .84 \\
\hline Male & 131 & 5 & 3.8 & & & \\
\hline Female & 68 & 3 & 4.4 & & & \\
\hline History of use of & & & & 982 & 2.07 to 46.50 & 004 \\
\hline Known & 14 & 3 & 21.4 & & & \\
\hline Not known & 185 & 5 & 2.7 & & & \\
\hline History of homo- & & & & & & \\
\hline $\begin{array}{l}\text { sexual activity } \\
\text { Known }\end{array}$ & 8 & 1 & 125 & 4.25 & .42 to 43.25 & .22 \\
\hline Not known & 123 & 4 & 3.3 & & & \\
\hline Total & 199 & 8 & 4.0 & - & - & - \\
\hline
\end{tabular}

${ }^{1}$ Odds ratios were based on two comparison groups: black and Hispanic versus Caucasian and Asian.

${ }^{2} \mathrm{~N}=131$ (males only)

the Western Blot and classified using to current recommendations of the Centers for Disease Control (1).

The group profile provided descriptive data. Antibody test results linked to patients' demographic characteristics and HIV risk behaviors provided prevalence rates within subgroups. All data were measured at either a nominal or an ordinal level, and contingency table analysis was used to interpret data. Chi square tests were performed to examine differences between groups. Unadjusted odds ratios were used to estimate relative risk for HIV serostatus. Unadjusted odds ratios estimate relative risk for each factor without controlling for effects of other risk variables. Adjusted odds ratios were calculated if unadjusted ratios were statistically significant. Risk estimates were derived from logistic regression coefficients.

Results

Blood specimens were obtained for HIV-1 antibody testing from 199 (84.3 percent) of 236 eligible pa- tients. Of the 199 tested specimens, eight were reactive to enzyme immunoassay, and all eight were confirmed antibody positive by Western Blot, for a seroprevalence of 4 percent. The distribution of positive results is shown in Table 1.

Of the three HIV-positive women, one had a history of injecteddrug use. Of the five HIV-positive men, two had a history of injecting drugs and one had used injected drugs and engaged in homosexual behavior. Of the 65 seronegative women, two (3.1 percent) had used injected drugs. Of the 126 seronegative men, nine ( 7.1 percent) used injected drugs and seven (5.6 percent) had a history of homosexual activity.

Relative risk estimators were derived from unadjusted odds ratios to compare the risks of testing positive for HIV-1 antibodies among the patients with and without identified risk factors. The unadjusted odds ratios showed that patients with a history of drug injection were almost ten times more likely to be seropositive than patients without such a history. No other variable showed a significant relationship to HIV-positive status. The strong relationship of injected-drug use and HIV status was maintained using multiple logistic regression analysis when age, ethnicity, and gender were controlled for (adjusted odds ratio $=9.87$, 95 percent confidence interval $=1.90$ to $51.27, \mathrm{p}=.006$ ).

\section{Discussion and conclusions}

Our expectation that long-stay patients would have lower rates of HIV infection than recently admitted patients was not supported. The HIV seroprevalence of 4 percent among long-stay patients is comparable to the 5.2 percent seroprevalence found among new admissions (1) and the 6.4 percent rate among hospitalized homeless patients removed from the street (10) and admitted to the same state hospital.

The only behavior significantly associated with HIV seropositivity among long-stay patients was use of injected drugs after 1978. However, information on sexual risk behavior is limited by the anonymous seroprevalence methodology, which depends on sexual risk behavior being noted in patients' charts (1). In fact, detailed interviews with patients on other units at the same hospital, unlinked to this anonymous seroprevalence study, established alarming rates of unsafe sexual activity among patients (Cournos F, McKinnon K, Meyer-Bahlburg $\mathrm{H}$, et al, unpublished manuscript, 1992).

That long-stay patients appear to have rates of HIV infection comparable to those of newly admitted patients may reflect either similar risk activity or the greater likelihood that acutely ill HIV-infected patients are difficult to discharge and will become long-stay patients, or both.

In conclusion, the same attention needs to be paid to the prevention, detection, and management of HIV illness in long-term psychiatric patients as in psychiatric patients admitted for acute care.

\section{Acknowledgments}

This study was funded by National Institute of Mental Health grant $\mathrm{MH}$ 46251 and the New York State Office of Mental Health. The authors thank 
Brenda Agosin, M.A., Steve Engelmann, Arthur Harewood, Wallace Nesby, Cathleen Norman, and Harbert Rice.

\section{References}

1. Cournos F, Empfield M, Horwath E, et al: HIV seroprevalence among patients admitted to two psychiatric hospitals. American Journal of Psychiatry 148: 1225-1230, 1991

2. Sacks M, Dermatis H, Looser-Ott S, et al: Seroprevalence of HIV and risk factors for AIDS in psychiatric inpatients. Hospital and Community Psychiatry 43:736-737, 1992

3. Dorwatt RA: A ten-year follow-up of the effects of deinstitutionalization. Hospital and Community Psychiatry 39:287-291, 1988

4. Herr BE, Abraham HD, Anderson W: Length of stay in a general hospital psychiatric unit. General Hospital Psychiatry
13:68-70, 1991

5. Richardson MA, Craig TJ, Jaugland G: Treatment patterns of young chronic schizophrenic patients in the era of deinstitutionalization. Psychiatric Quarterly 57:104-110, 1985

6. Modestin J: Patterns of overt sexual interaction among acute psychiatric inpatients. Acta Psychiatrica Scandinavica 64:446-459, 1981

7. Keitner G, Grof P: Sexual and emotional intimacy between psychiatric inpatients: formulating a policy. Hospital and Community Psychiatry 32:188-193, 1981

8. Binder RL: Sex between psychiatric inpatients. Psychiatric Quarterly 57:121126,1985

9. Welch S, Meagher J, Soos J, et al: Sexual behavior of hospitalized chronic psychiatric patients. Hospital and Community Psychiatry 42:855-856, 1991

10. Empfield M, Cournos F, Meyer I, et al: HIV seroprevalence among homeless patients admitted to a psychiatric inpatient unit. American Journal of Psychiatry 150:47-52, 1993

\section{Medical Findings in School-Age Psychiatric Inpatients Grouped by Public and Private Payment}

\section{Richard Dalton, M.D. Jorge H. Daruna, Ph.D. Cynthia D. Strecker, M.S.}

The few studies that have examined the results of laboratory tests and physical examinations during psychiatric hospitalization of adults (1), adolescents (2), and children (3) have found them of little use because they uncover very few medical disorders. Somatic complaints of latency-age inpatients have usually been shown at follow-up not to be a manifestation of medical illness (4).

During the past few years, however, it has been our clinical impression that the more economically and

The authors are affiliated with the department of psychiatry and neurology at Tulane University Medical Center, 1430 Tulane Avenue, New Orleans, Louisiana 70112. socially deprived patients on our psychiatric inpatient unit for latencyage children, namely those in state custody, have had an unusually high number of symptoms and physical illnesses that were discovered during their hospitalization. We thus decided to further evaluate the medical status of our patients and the clinical utility of medical workups for these patients.

This study was designed to determine whether during the last three years patients on our unit whose care was subsidized by state medical assistance had a greater number of positive medical findings than patients with private insurance as reflected in the findings of physical examinations, laboratory tests, and subspecialty consultations.

\section{Methods}

The study was a retrospective review of hospital records of patients admitted to our previously described (5) inpatient psychiatric unit for schoolage children (ages five to 12). The last 50 privately insured patients and the last 50 patients subsidized by state medical assistance who were consecutively admitted in the period between 1989 and 1991 were included in the study.

The inpatient medical workup consisted of a thorough medical history and physical examination within 24 hours of admission. Routinely ordered laboratory tests included a complete blood count with a differential cell count, a urinalysis, and a Chem-12 profile (sodium, potassium, glucose, creatinine, total protein, albumin, calcium, phosphate, total bilirubin, alkaline phosphatase, serum glutamic oxalotransaminase, and serum glutamic pyruvate transaminase). Based on the results of the initial workup, clinicians requested consultation with a hospital-based pediatric subspecialty or ordered specialized tests (computerized tomography, electroencephalogram, electrocardiogram, lead level, thyroid panel, chromosomal analysis, drug test, magnetic resonance imaging, timed urine chemistries, blood culture, liver profile, and platelet function screen). Such tests were also ordered when symptoms of unknown origin or symptoms indicative of the need for medical treatment developed during hospitalization (for example, vision and hearing complaints not previously discovered or high fever).

In addition to the patients' age, gender, and psychiatric diagnosis, data on medical history, past medical hospitalization, and previous psychiatric hospitalization were obtained. (Race or ethnicity was not a variable that was examined because fewer than five of the children were from minority groups.) The results of the physical examinations were reviewed for the number of positive findings (that is, signs not expected during a normal examination) as well as the number of positive findings not previously known. The results of the complete blood count with a differential cell count, the urinalysis, and the Chem-12 were also reviewed for findings outside the normal clinical 